\title{
C4d as a Screening Tool and an Independent Predictor of Clinical Outcomes in Lupus Nephritis and IgA Nephropathy
}

\begin{abstract}
Xiaoqian Yang ${ }^{\dagger}$, Yanhong Yuan ${ }^{\dagger}$, Xinghua Shao, Huihua Pang, Xiajing Che, Liou Cao, Minfang Zhang, Yao Xu, Zhaohui Ni, Chaojun Qi*, Qin Wang* and Shan Mou*
\end{abstract}

Department of Nephrology, Renji Hospital, School of Medicine, Shanghai Jiao Tong University, Shanghai, China

\section{OPEN ACCESS}

Edited by:

Cheng Yang,

Fudan University, China

Reviewed by:

Takaharu Ichimura,

Brigham and Women's Hospital and

Harvard Medical School,

United States

Li Li,

Thomas Jefferson University,

United States

*Correspondence:

Chaojun Q

qichaojun77@126.com

Qin Wang

qinwang_1975@126.com

Shan Mou

shan_mou@shsmu.edu.cn

†These authors have contributed equally to this work

Specialty section:

This article was submitted to Nephrology,

a section of the journal

Frontiers in Medicine

Received: 10 December 2021 Accepted: 03 January 2022

Published: 31 January 2022

Citation:

Yang $X$, Yuan $Y$, Shao $X$, Pang $H$, Che $X$, Cao L, Zhang M, Xu Y, Ni Z, Qi C, Wang Q and Mou S (2022) C4d

as a Screening Tool and an

Independent Predictor of Clinical Outcomes in Lupus Nephritis and IgA Nephropathy. Front. Med. 9:832998.

doi: 10.3389/fmed.2022.832998
Background: As an indispensable marker of complement cascades activation, C4d was confirmed of its crucial role in the pathogenesis of both lupus nephritis (LN) and IgA nephropathy (IgAN). While the studies directly comparing the diagnostic value, and outcomes predicting function of $\mathrm{C} 4 \mathrm{~d}$ between $\mathrm{LN}$ and IgAN are still absent.

Methods: A cohort of 120 LN patients, 120 IgAN patients who were diagnosed by renal biopsy between January 2015 and December 2017 and 24 healthy age matched controls were prospectively analyzed. The patients were followed till December 2020. The outcomes were adverse disease treatment response (disease relapse) and kidney disease progression event (decline of estimated glomerular filtration rate by more than $20 \%$ or end-stage kidney disease). The renal C4d deposition proportion and pattern were compared between IgAN and LN patients. In addition, the relationship between renal C4d deposition and disease subtypes, disease relapse as well as disease progression for $L N$ and IgAN patients were also analyzed.

Results: The LN, IgAN patients and healthy controls were well matched in ages. The follow-up period was 38.5 (30.3-60.8) months for $L N$ patients and 45.0 (30.5-57.0) months for IgAN patients. 78 patients (65.0\%) with $\mathrm{LN}$ had renal C4d deposition, compared with only 39 IgAN patients (32.5\%) with C4d deposition in renal tissues $(P<0.001)$. The LN patients shared different renal C4d distribution patterns with IgAN patients. Compared with IgAN patients, the C4d deposition in LN patients was significantly more in renal glomerulus $(P<0.001)$ and less in renal tubules $(P=0.003)$. For disease subtypes, renal C4d deposition was especially strong in class $V$ membranous $\mathrm{LN}$ and IgAN with tubulointerstitial fibrosis (T1/T2) lesions. Renal C4d deposition was independently correlated with the disease relapse of $L N$ patients $(H R=1.007, P$ $=0.040$ ), and acted as an independent predictor of disease progression during the follow-up period for IgAN patients $(H R=1.821, P=0.040)$.

Conclusions: Renal C4d distribution proportion and pattern differed between $L N$ and IgAN patients. The presence of C4d in renal tissue acted as an independent predictor of relapse for $L N$ patients and disease progression for IgAN patients.

Keywords: complement, C4d, lupus nephritis, IgA nephropathy, relapse, disease progression 


\section{INTRODUCTION}

Systemic lupus erythematosus (SLE) is an autoimmune disease, and up to $60 \%$ of SLE patients developed Lupus nephritis (LN) during the course of the disease (1). Immune complex deposition and complement activation in the kidney significantly contributed to the pathogenesis of $\mathrm{LN}$ (2-4). IgA nephropathy (IgAN) is the most common primary glomerulonephritis worldwide, and the diagnostic histologic hallmark for IgAN is dominant or codominant IgA staining on kidney biopsy (5). Complement activation through alternative and lectin pathways might play significant roles in the pathogenesis of IgAN via upregulating local inflammatory responses and glomerular injury $(6,7)$.

Although both LN and IgAN are immune complex-mediated diseases, the pathogenesis, their etiologies, and the therapeutic strategies are different. However, co-occurrences of SLE and IgAN were found in some patients (8-10). Shared genetics between immune-related diseases may account for this in some extent (11). In this case, it is significant to find a reliable screening and diagnostic tool to differentiate the renal disease in SLE patients between LN and IgAN, which has important implications on therapeutic regimens to be adopted, and further influences the disease prognosis in both short and long term.

Dysfunction of the complement system plays a crucial role in the development of $\operatorname{LN}$ and $\operatorname{IgAN}(6,12)$. As the most polymorphic protein in complement system, C4d is considered as an indispensable marker of complement cascades activation (13). Meanwhile, C4d has a much longer half-life, and appears to persist for weeks in the tissue due to covalent binding to the tissue structures (14), indicating its great potential for the clinical application as a marker for the progression of LN and IgAN. Previous studies indicated that renal C4d deposition in LN is associated with the LN subtype, the immune complex deposits, and the glomerular microthrombosis $(15,16)$. For IgAN, C4d deposition in renal tissue has been associated with aggressive histology and poor renal outcome $(17,18)$. However, studies specific for comparing the roles of C4d in LN and IgAN are still absent. Based on the limitations of the existing studies, the aim of our study is to compare the screening and diagnostic value, meanwhile, the clinical and prognostic implications of renal C4d deposition between LN and IgAN patients.

\section{MATERIALS AND METHODS}

\section{Study Population}

Patients who were diagnosed of LN or IgAN by renal biopsy between January 2015 and December 2017 from the Department of Nephrology, Ren Ji Hospital, Shanghai, China were included in the study. The exclusion criteria were as follows: (1) age less than 18 years; (2) LN or IgAN of transplant kidney; (3) co-occurrence of severe systemic disease that may affect the prognosis (such as heart failure, malignant tumor); (4) lost to follow-up. The patients had regular follow-up visits till December 2020 at intervals of at least 3-6 months. And the age matched controls were healthy blood donors without indicators of renal disease. Finally a total of 264 subjects were enrolled, including
$120 \mathrm{LN}$ patients, 120 IgAN patients and 24 healthy controls. All the data were collected prospectively.

\section{Outcomes}

The analysis included disease treatment and disease progression outcomes. Adverse disease treatment outcome was disease relapse at the sixth month after the initiation of therapy. The disease progression event was defined as a decline of eGFR by more than $20 \%$ or end-stage kidney disease during the followup period. End-stage kidney disease was defined as eGFR $<15$ $\mathrm{mL} / \mathrm{min} / 1.73 \mathrm{~m}^{2}$ or initiation of renal replacement therapy.

\section{Definitions}

The treatment response was evaluated at the sixth month after the initiation of therapy for LN and IgAN patients. In the LN patients, the response criteria were defined according to the KIDGO guideline criteria (19). Complete response (CR): Return of serum creatinine (Scr) to previous baseline, plus a decline in the urine protein-creatinine ratio (uPCR) to $<500 \mathrm{mg} / \mathrm{g}$ $(<50 \mathrm{mg} / \mathrm{mmol})$. Partial response (PR): Stabilization $( \pm 25 \%)$, or improvement of Scr, but not to normal, plus a $<50 \%$ decrease in uPCR. If there was nephrotic-range proteinuria ( $\mathrm{uPCR} \geq 3,000 \mathrm{mg} / \mathrm{g}[\geq 300 \mathrm{mg} / \mathrm{mmol}]$ ), improvement requires a $\geq 50 \%$ reduction in $\mathrm{uPCR}$, and a $\mathrm{uPCR}<3,000 \mathrm{mg} / \mathrm{g}[<300$ $\mathrm{mg} / \mathrm{mmol}$. Non-response (NR): Fail to meet any of the criteria for remission. And the relapse criteria were defined according to the ACR 2006 clinical trial criteria (20). Disease relapse was characterized as a $>25 \%$ increase in the Scr, or a $50 \%$ or more increase in proteinuria or active urine sediment characterized by $>5$ erythrocytes/HPF and/or cellular casts.

In the IgAN patients, $\mathrm{CR}$ was defined as a urine protein excretion $(\mathrm{UPE})<0.3 \mathrm{~g} / \mathrm{d}$, along with normalization of all biochemical findings and a lack of worsening of renal function at the sixth month; PR was defined as at least a $50 \%$ reduction in UPE at the sixth month compared with baseline; NR was defined as a $<50 \%$ reduction in UPE or an increase in UPE with or without renal deterioration after receiving 6 months of therapy; Relapse was defined as the reappearance of significant proteinuria, defined as $>1.0 \mathrm{~g} / \mathrm{d}$ and as a UPE increase of $50 \%$ from the lowest level of proteinuria after the start remission (21).

The estimated glomerular filtration rate (eGFR) was calculated with modified MDRD equation for Chinese patients:eGFR = 186 $\times(\mathrm{Scr} / 88.4)^{-1.154} \times \mathrm{age}^{-0.203}(\times 0.742$, female $)(22)$.

\section{Evaluation of Renal Biopsies}

Renal biopsies of LN patients enrolled in this study were obtained and evaluated using the International Society of Nephrology/Renal Pathology Society (ISN/RPS) classification (23), and IgAN patients' renal biopsies were evaluated using the Oxford classification (24).

C4d staining was performed at Ren Ji Hospital by one of the investigators, who were blinded to the identity of the tissue. Biopsy samples were fixed in $4 \%$ paraformaldehyde (4\% PFA) solution and embedded in paraffin. Paraffin sections ( $4 \mu \mathrm{m}$ thick) were placed on positively charged slides and kept in a stove at $60^{\circ} \mathrm{C}$ for $1 \mathrm{~h}$. Sections were deparaffinized and rehydrated through a series of xylene and graded alcohols. Antigen retrieval 


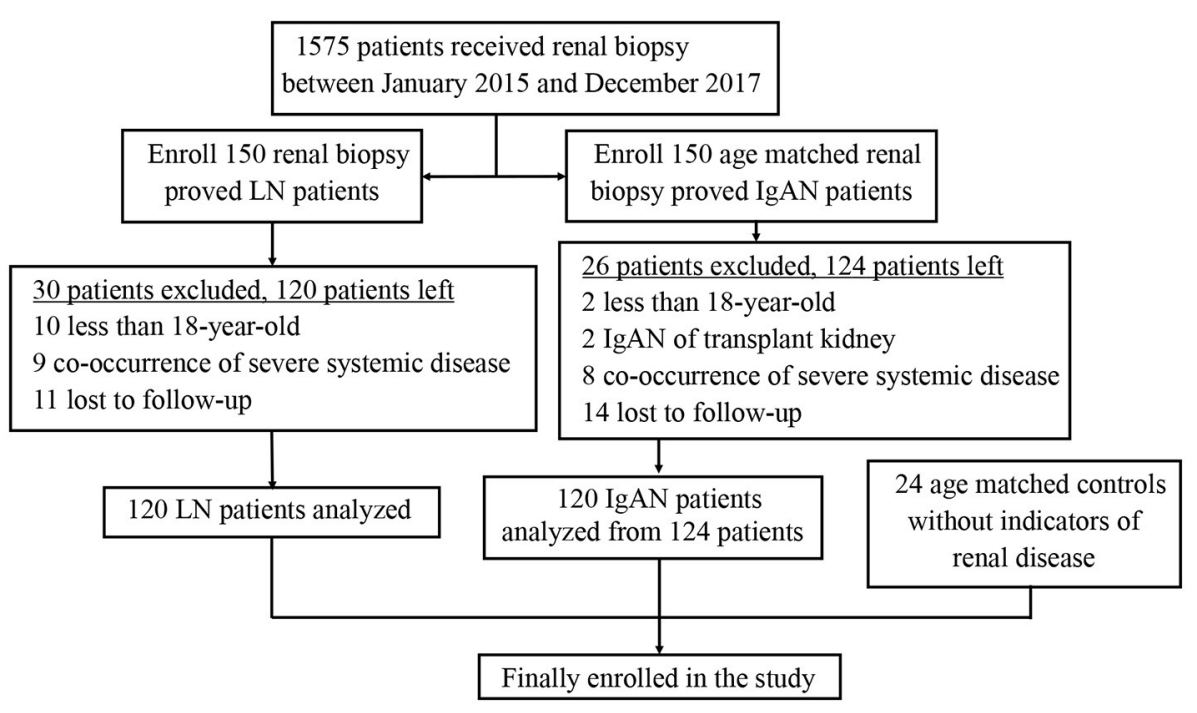

FIGURE 1 | Flow diagram of the study design. A total of $120 \mathrm{LN}$ patients, 120 IgAN patients and 24 healthy controls were enrolled into the study. LN, lupus nephritis; IgAN, IgA nephropathy.

was performed by autoclave in a high-pressure sterilizer at $121^{\circ} \mathrm{C}$ for $5 \mathrm{~min}$ in $10 \mathrm{mM}$ citrate buffer ( $\mathrm{pH}$ 6.0). Endogenous peroxidase was blocked in $3 \% \mathrm{H}_{2} \mathrm{O}_{2}$ for $10 \mathrm{~min}$. The primary mouse anti-human C4d monoclonal antibody (LP69; Abcam) was applied at a dilution of 1:50 in primary antibody diluent (ZCIB01), and slides were incubated overnight at $4{ }^{\circ} \mathrm{C}$. The slides were then incubated with goat anti-mouse IgG antibody (115-035-003; Jackson) for $1 \mathrm{~h}$, and staining was visualized with the DAB (D8001; Sigma) for $5 \mathrm{~min}$. Sections were washed with PBS ( $\mathrm{pH}$ 7.4) between each step ( 3 times for 5 min each time). Finally, sections were counterstained with Mayer's hematoxylin, air-dried, cleared in xylene, and covered with glasses. Paraffin sections ( $4 \mu \mathrm{m}$ thick) were also stained with periodic acid Schiff (PAS) and periodic acid-silver methenamine (PASM). Sections were imaged on a light microscope.

\section{Urine and Serum C4d Measurements}

The serum and urine samples were collected soon after admission to the hospital. The collected samples were centrifuged at 3,000 g for $10 \mathrm{~min}$ to remove cellular debris. We aliquoted serum and urine supernatant into bar-coded cry-vials and stored the samples at $-80^{\circ} \mathrm{C}$ until biomarker measurement. No additives or protease inhibitors were added.

We measured the serum and urine biomarker C4d with a commercially available ELISA kit (Dako, Denmark) following manufacturer's instruction. We measured urine creatinine by the modified Jaffe reaction. Personnel performing the biomarker measurements were blinded to each patient's clinical information. All biomarkers were measured from frozen aliquots that did not undergo any additional freeze-thaw cycles.

\section{Statistical Analysis}

The statistical analysis was completed with SPSS, version 26.0. The normally distributed variables were expressed as the means $\pm \mathrm{SD}$ and compared using a $t$-test or analysis of variance (ANOVA) as required. The non-parametric variables were expressed as the medians with $25^{\text {th }}$ and $75^{\text {th }}$ percentiles and compared using the Mann-Whitney $\mathrm{U}$ test. The Chi-square test was employed for the categorical variables. Cox proportional hazards regression was conducted with the candidate variables. The multivariable regression analysis was performed at the 0.1 level of significance for variables from a univariable analysis and used a stepwise forward selection procedure (25). Cumulative survival curves were derived using the Kaplan-Meier method, and the differences between survival curves were compared using the log-rank test.

\section{RESULTS}

\section{Baseline Clinical Characteristics and Histologic Findings}

A total of 264 subjects were finally included in the study cohort, including $120 \mathrm{LN}$ patients, $120 \mathrm{IgAN}$ patients and 24 healthy controls (Figure 1). Baseline characteristics of all participants were listed in Table 1. Subjects from all groups were matched in ages. There was no difference in follow-up duration between LN patients [38.5(30.3-60.8) months] and IgAN patients [45.0(30.557.0) months] $(P>0.05)$. Compared with IgAN patients, $\mathrm{LN}$ patients had lower serum C3 levels $[1.10(0.96-1.25) \mathrm{g} / \mathrm{L}$ vs. 0.37 (0.29-0.53) g/L, $P<0.05]$ and lower serum C4 levels [0.24(0.21$0.29) \mathrm{g} / \mathrm{L}$ vs. $0.08(0.02-0.29) \mathrm{g} / \mathrm{L}, P<0.05]$. Besides, $\mathrm{LN}$ patients with C4d deposition in renal tissue were much more than IgAN patients $(65.0 \%$ vs. $32.5 \%, P<0.001)$. Patients with $\mathrm{LN}$ and IgAN showed comparable levels of serum C4d [23.60 (19.52$25.94) \mu \mathrm{g} / \mathrm{ml}$ vs. $23.43(19.37-25.93) \mu \mathrm{g} / \mathrm{ml}, P>0.05]$ and urine C4d excretion [18.34 (15.59-25.05) $\mu \mathrm{g} / \mathrm{ml}$ vs. 26.04(22.90-29.35) $\mu \mathrm{g} / \mathrm{ml}, P>0.05]$. 
TABLE 1 | Clinical and histologic characteristics at baseline for LN, IgAN and healthy control patients.

\begin{tabular}{|c|c|c|c|}
\hline Characteristics & $\begin{array}{l}\text { Healthy } \\
\text { control } \\
(N=24)\end{array}$ & $\begin{array}{c}\text { LN } \\
(N=120)\end{array}$ & $\begin{array}{l}\operatorname{IgAN}(N \\
=120)\end{array}$ \\
\hline Age (years) & $\begin{array}{c}36.0 \\
(23.5-50.4)\end{array}$ & $35.9(20.3-55.5)$ & $\begin{array}{c}32.0 \\
(21.6-45.1)\end{array}$ \\
\hline $\begin{array}{l}\text { Gender: Female, } \\
n(\%)\end{array}$ & $12(50.0)$ & $108(90.0)$ & $64(53.3)$ \\
\hline $\begin{array}{l}\text { Follow-up } \\
\text { (months) }\end{array}$ & - & $38.5(30.3-60.8)$ & $\begin{array}{c}45.0 \\
(30.5-57.0)\end{array}$ \\
\hline $\mathrm{Hb}(\mathrm{g} / \mathrm{L})$ & $\begin{array}{c}132.0(122.5- \\
145.5)\end{array}$ & $\begin{array}{c}101.0(86.5- \\
115.5)\end{array}$ & $\begin{array}{c}139.0 \\
(128.5-151.5)\end{array}$ \\
\hline $\operatorname{Scr}(\mu \mathrm{mol} / \mathrm{L})$ & $\begin{array}{c}60.2 \\
(50.0-85.1)\end{array}$ & $62.0(52.2-80.3)$ & $\begin{array}{c}88.0 \\
(60.0-111.6)\end{array}$ \\
\hline $\begin{array}{l}\text { eGFR } \\
\left(\mathrm{ml} / \mathrm{min} / 1.73 \mathrm{~m}^{2}\right)\end{array}$ & $\begin{array}{c}105.9 \\
(90.5-120.5)\end{array}$ & $\begin{array}{c}105.6 \\
(69.7-141.5)\end{array}$ & $\begin{array}{c}85.8 \\
(60.3-110.7)\end{array}$ \\
\hline $\begin{array}{l}\text { Serum albumin } \\
(\mathrm{g} / \mathrm{L})\end{array}$ & $\begin{array}{c}39.5 \\
(38.6-45.2)\end{array}$ & $33.6(25.6-41.6)$ & $\begin{array}{c}38.5 \\
(35.7-44.2)\end{array}$ \\
\hline $\begin{array}{l}\text { anti-dsDNA } \\
\text { antibody (IU/mL) }\end{array}$ & - & $34.3(10.1-69.3)$ & - \\
\hline uRBC (/HP) & - & $\begin{array}{c}21.0 \\
(15.6-100.2)\end{array}$ & $\begin{array}{c}15.0 \\
(8.1-143.5)\end{array}$ \\
\hline UPE (g/d) & - & $1.38(0.27-4.58)$ & $\begin{array}{c}1.05 \\
(0.81-3.54)\end{array}$ \\
\hline $\begin{array}{l}\text { Serum C3 level } \\
(\mathrm{g} / \mathrm{L})\end{array}$ & - & $0.37(0.29-0.53)$ & $\begin{array}{c}1.10 \\
(0.96-1.25)\end{array}$ \\
\hline $\begin{array}{l}\text { Serum C4 level } \\
(\mathrm{g} / \mathrm{L})\end{array}$ & - & 0.08(0.02-0.29) & $0.24(0.21-0.29)$ \\
\hline $\begin{array}{l}\text { Serum C4d level } \\
(\mu \mathrm{g} / \mathrm{ml})\end{array}$ & $\begin{array}{c}19.90 \\
(15.07-23.33)\end{array}$ & $\begin{array}{c}23.60 \\
(19.52-25.94)\end{array}$ & $\begin{array}{c}23.43 \\
(19.37-25.93)\end{array}$ \\
\hline $\begin{array}{l}\text { Urine C4d } \\
\text { excretion }(\mu \mathrm{g} / \mathrm{ml})\end{array}$ & $\begin{array}{c}16.23 \\
(14.56-24.22)\end{array}$ & $\begin{array}{c}18.34 \\
(15.59-25.05)\end{array}$ & $\begin{array}{c}26.04 \\
(22.90-29.35)\end{array}$ \\
\hline \multicolumn{2}{|c|}{ Renal biopsy, classification } & $\begin{array}{c}\text { ISN/RPS } \\
\text { pathologic } \\
\text { classification, } n \\
(\%)\end{array}$ & $\begin{array}{c}\text { Oxford } \\
\text { classification, } n \\
(\%)\end{array}$ \\
\hline & - & III, 20 (1.7) & M1, 70 (58.3) \\
\hline & - & IV, 53 (44.2) & $\mathrm{E} 1,30(25.0)$ \\
\hline & - & $I I+\mathrm{V}, 6(5.0)$ & S1, $89(74.2)$ \\
\hline & - & IV+V, 19 (15.8) & $\begin{array}{c}\text { T0/T1/Т2, } 68 \\
(56.7) / 35 \\
(29.2) / 17(14.2)\end{array}$ \\
\hline & - & V, 22 (18.3) & $\begin{array}{c}\mathrm{C} 0 / \mathrm{C} 1 / \mathrm{C} 2,62 \\
(51.7) / 53 \\
(44.2) / 5(4.2)\end{array}$ \\
\hline \multicolumn{4}{|c|}{ C4d deposition in renal tissue } \\
\hline $\begin{array}{l}\text { C4d positive, } n \\
(\%)\end{array}$ & - & $78(65.0)$ & $39(32.5)$ \\
\hline
\end{tabular}

The median $\left(25^{\text {th }}-75^{\text {th }}\right.$ percentile) was shown if the variables did not show normal distributions, and $n$ (\%) was shown for the categorical variables. LN, lupus nephritis; IgAN, IgA nephropathy; Hb, hemoglobin; Scr, serum creatinine; eGFR, estimated glomerular filtration rate; $U R B C$, urine red blood cell; UPE, urine protein excretion.

\section{Renal C4d Deposition Patterns in LN and IgAN Patients}

C4d deposits could be found in glomerulus, tubules, interstitium and peritubular capillaries of renal tissue for both LN and IgAN patients. For LN patients, most of the C4d deposited
TABLE 2 | The C4d distribution patterns in renal tissue for $L N$ and IgAN patients.

\begin{tabular}{lccc}
\hline C4d distribution in renal tissue & LN $(\boldsymbol{n}=\mathbf{7 8})$ & $\operatorname{IgAN}(\boldsymbol{n}=\mathbf{3 9 )}$ & $\boldsymbol{P}$ value \\
\hline Glomerulus, $n(\%)$ & $72(92.3)$ & $22(56.4)$ & $<0.001$ \\
Tubules, $n(\%)$ & $7(9.0)$ & $12(30.8)$ & 0.003 \\
Arterioles, $n$ (\%) & $4(5.1)$ & $3(7.7)$ & 0.890 \\
Peritubular capillaries, $n(\%)$ & $15(19.2)$ & $6(15.4)$ & 0.609
\end{tabular}

$P$ value: comparation between $L N$ and IgAN group. $L N$, lupus nephritis; IgAN, IgA nephropathy.

in glomerulus (92.3\%) and peritubular capillaries (19.2\%) of renal tissue. For IgAN patients, most of C4d deposited in renal glomerulus (56.4\%) and tubules (30.8\%). Although both LN and IgAN patients had the most C4d distribution in renal glomerulus, the glomerular C4d deposition in $\mathrm{LN}$ patients was significantly more than IgAN patients $(\mathrm{P}<0.001)$. On the contrary, the LN patients had statistically less $\mathrm{C} 4 \mathrm{~d}$ deposition in renal tubules compared with IgAN patients $(P=0.003)$ (Table 2, Figure 2).

C4d deposition were detected in various subtypes of LN but especially strong in class $\mathrm{V}$ membranous LN. Accordingly, IgAN patients with renal C4d deposition had a greater probability of tubulointerstitial fibrosis (T1/T2). While other Oxford score indices including mesangial hypercellularity (M1), endothelial hypercellularity (E1) lesions, segmental (S1) lesions and crescents (C1/C2) lesions had no significant correlations with renal C4d deposition (Table 3, Figure 3).

\section{Correlation Between Renal C4d Deposition With Clinical Outcomes for LN and IgAN Patients}

For disease treatment responses, it is indicated that $\mathrm{LN}$ patients with renal C4d deposition had less probability of sustainedremission $(P=0.002)$ and higher risk of relapse $(P=$ 0.001) compared with $\mathrm{C} 4 \mathrm{~d}$ negative patients. While there was no significant relationship between renal C4d deposition with relapse for IgAN patients $(P=0.052)$. For sustained remission, C4d deposited IgAN patients were statistically less in comparison to C4d negative IgAN patients $(P=0.043)$ (Table 4). As far as the renal function outcome during the follow-up period, IgAN patients with renal C4d deposition were associated with more IgAN progression events compared with C4d negative ones $(P<0.001)$. For LN patients, the presence of renal $\mathrm{C} 4 \mathrm{~d}$ deposition was not statistically correlated with $\mathrm{LN}$ progression during the follow-up time $(P=0.812)$ (Table 5).

\section{C4d as an Independent Predictor for Clinical Outcomes of LN and IgAN Patients}

To further explore the correlation between renal C4d deposition with disease relapse of $\mathrm{LN}$ patients, renal C4d deposition and other variables were included into the univariate and multivariate Cox regression analysis and concluded that high baseline serum 


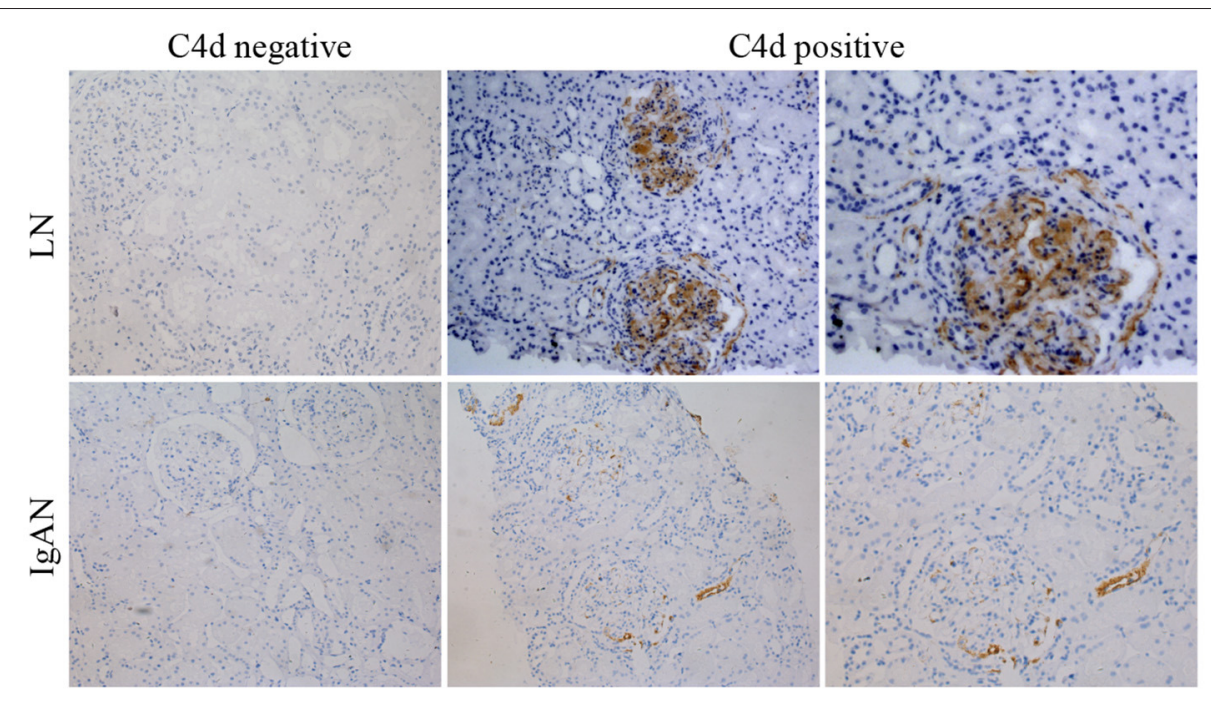

FIGURE 2 | Representative immunohistochemical images showing negative or positive C4d deposition in renal tissue of LN and IgAN patients. The top panels were images for LN patients with negative (left panel) or positive (central and right panel) C4d deposition. The bottom panels were images for lgAN patients with negative (left panel) or positive (central and right panel) C4d deposition. $\times 200$ (left and central panels). $\times 400$ (right panels). LN, lupus nephritis; IgAN, IgA nephropathy.

TABLE 3 | Relationship between renal C4d deposition with the ISN/RPS pathologic classes for $L N$ and the Oxford classification for IgAN.

\begin{tabular}{llll}
\hline Pathologic classification & C4d positive & C4d negative & $P$ value \\
\hline LN (ISN/RPS) & $\mathbf{N}=\mathbf{7 8}$ & $\mathbf{N}=\mathbf{4 2}$ & \\
III, $n$ (\%) & $10(12.82)$ & $10(23.81)$ & 0.123 \\
IV, $n$ (\%) & $30(38.46)$ & $23(54.76)$ & 0.086 \\
III+V, $n$ (\%) & $3(3.85)$ & $3(7.14)$ & 0.725 \\
IV+V, $n$ (\%) & $15(19.23)$ & $4(9.52)$ & 0.165 \\
V, $n$ (\%) & $20(25.64)$ & $2(4.76)$ & 0.005 \\
IgAN (Oxford-MESTC score) & $\mathbf{N = 3 9}$ & $\mathbf{N}=\mathbf{8 1}$ & \\
Mesangial proliferation (M1), $n$ & $24(61.5)$ & $46(56.8)$ & 0.621 \\
(\%) & & & \\
Endocapillary proliferation (E1), $n$ & $11(28.2)$ & $19(23.5)$ & 0.574 \\
(\%) & & & \\
Segmental sclerosis (S1), $n(\%)$ & $31(79.5)$ & $58(71.6)$ & 0.356 \\
Interstitial fibrosis/Tubular & $22(56.4)$ & $30(37.0)$ & 0.045 \\
atrophy (T1-T2), $n$ (\%) & & & 0.654 \\
Crescent (C1-C2), $n$ (\%) & $20(51.3)$ & $38(46.9)$ & \\
\hline P & &
\end{tabular}

$P$ value: comparation between C4d positive and C4d negative group. LN, lupus nephritis; IgAN, IgA nephropathy.

anti-dsDNA antibody $(\mathrm{HR}=1.105, P=0.034)$ and renal $\mathrm{C} 4 \mathrm{~d}$ deposition ( $\mathrm{HR}=1.007, P=0.040)$ are independent predictors of disease relapse for LN patients (Table 6).

As far as the long-term renal function outcome for IgAN patients, univariate and multivariate Cox regression analysis suggested that high baseline serum creatinine (HR $=1.010, P=0.034)$ and renal $\mathrm{C} 4 \mathrm{~d}$ deposition $(\mathrm{HR}=$ 1.821, $P=0.040$ ) are independent predictors of disease progression during the follow-up period for IgAN patients (Table 7). Similarly, Kaplan-Meier plot also indicated that renal $\mathrm{C} 4 \mathrm{~d}$ deposition is positive correlated with disease progression by time for IgAN patients (log-rank test, $P<0.001$, Figure 4).

\section{DISCUSSION}

The complement system plays significant roles in the pathogenesis of $\operatorname{LN}$ and $\operatorname{IgAN}(6,12)$. As the complementsplit product and a good marker of classical and lectin pathway activation, C4d is usually used to monitor humoral rejection in renal transplant biopsy samples (26). Several studies have confirmed that $\mathrm{C} 4 \mathrm{~d}$ is associated with the pathogenesis of both LN and IgAN (15-18). Nevertheless, studies directly comparing the screening and diagnostic value, and outcomes predicting function of renal $\mathrm{C} 4 \mathrm{~d}$ deposition between $\mathrm{LN}$ and IgAN are still relatively sparse. In the present study, we demonstrated that LN and IgAN patients had different $\mathrm{C} 4 \mathrm{~d}$ deposition proportions and patterns in renal tissue. Moreover, renal C4d deposition was especially strong in class $\mathrm{V}$ membranous $\mathrm{LN}$ and IgAN with tubulointerstitial fibrosis lesions. Furthermore, the renal C4d deposition was an independent predictor of disease relapse for $\mathrm{LN}$ patients and disease progression for IgAN patients respectively.

Among many immune related renal diseases, $\mathrm{LN}$ and IgAN are the two most common glomerular diseases (27). As the frequent complication of SLE, LN involves multiple pathogenic pathways including aberrant apoptosis, autoantibody production, immune complex deposition and complement activation. The classical ISN/RPS classification system of LN is mainly based on glomerular lesions. While recently studies indicated that tubulointerstitial lesions and vascular injury were also important indicators of $\mathrm{LN}$ and should be incorporated into the classification of $\mathrm{LN}$ (28). IgAN is defined by the presence of IgA-dominant or co-dominant 
A

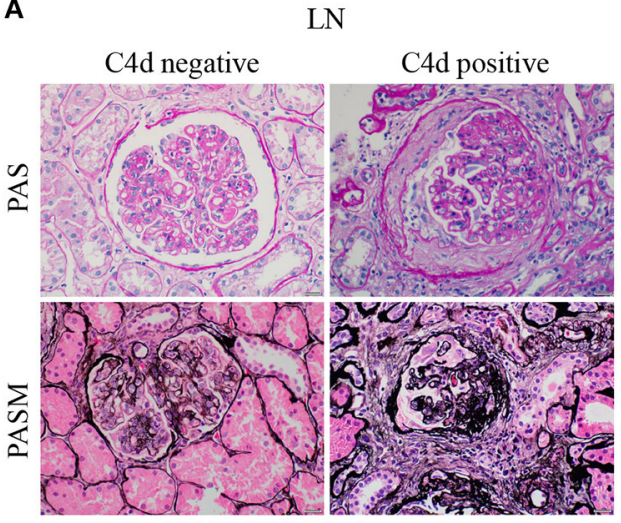

B

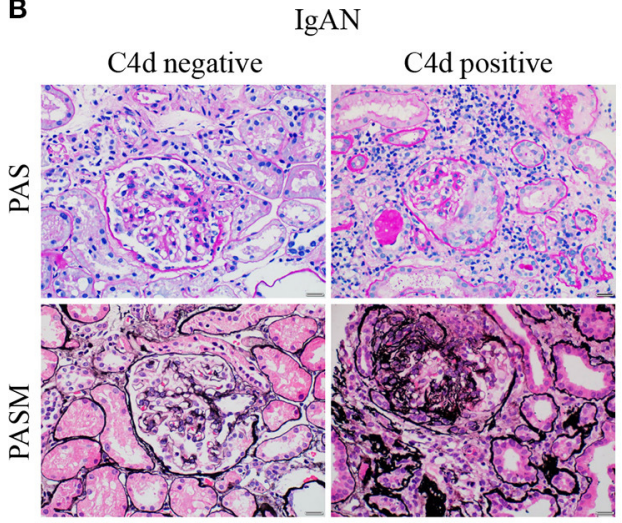

FIGURE 3 | Representative periodic acid schiff (PAS) and periodic acid-silver methenamine (PASM) images of LN and IgAN patients with negative or positive renal C4d deposition. (A) The representative PAS (top panels) and PASM (bottom panels) images of LN patients with (right panels) or without (left panels) renal C4d deposition. (B) The representative PAS (top panels) and PASM (bottom panels) images of IgAN patients with (right panels) or without (left panels) renal C4d deposition. Scale bars: $20 \mu \mathrm{m}$. LN, lupus nephritis; IgAN, IgA nephropathy; PAS, Periodic Acid Schiff; PASM, periodic acid-silver methenamine.

TABLE 4 | Relationship between renal C4d deposition with responses to treatment of $L N$ and $\operatorname{lgAN}$ patients.

\begin{tabular}{lccc}
\hline Responses to treatment & C4d positive & C4d negative & P value \\
\hline LN & $\mathbf{N}=\mathbf{7 8}$ & $\mathbf{N}=\mathbf{4 2}$ & \\
Sustained-remission, $n(\%)$ & $36(46.15)$ & $32(76.19)$ & 0.002 \\
Non-response, $n$ (\%) & $12(15.38)$ & $6(14.29)$ & 0.872 \\
Relapse, $n$ (\%) & $30(38.46)$ & $4(9.52)$ & 0.001 \\
IgAN & $\mathbf{N}=\mathbf{3 9}$ & $\mathbf{N}=\mathbf{8 1}$ & \\
Sustained-remission, $n(\%)$ & $24(61.54)$ & $64(79.01)$ & 0.043 \\
Non-response, $n$ (\%) & $6(15.38)$ & $9(11.11)$ & 0.713 \\
Relapse, $n$ (\%) & $9(23.07)$ & $8(9.87)$ & 0.052 \\
\hline P value: comparation between C4d positive and C4d negative group. LN, lupus nephritis; \\
IgAN, IgA nephropathy.
\end{tabular}

TABLE 5 | Relationship between renal C4d deposition with the disease progression of LN and IgAN patients during the follow-up period.

\begin{tabular}{lccc}
\hline Disease progression & C4d positive & C4d negative & $\boldsymbol{P}$ value \\
\hline LN & $\mathbf{N}=\mathbf{7 8}$ & $\mathbf{N}=\mathbf{4 2}$ & \\
LN progression event, $\mathrm{n}(\%)$ & $28(35.89)$ & $16(38.09)$ & 0.812 \\
IgAN & $\mathbf{N}=\mathbf{3 9}$ & $\mathbf{N}=\mathbf{8 1}$ & \\
IgAN progression event, $\mathrm{n}(\%)$ & $30(76.92)$ & $7(8.64)$ & $<0.001$
\end{tabular}

$P$ value: comparation between $C 4 d$ positive and $C 4 d$ negative group. $L N$, lupus nephritis; IgAN, IgA nephropathy.

immune deposits within glomeruli. The pathogenesis of IgAN is a multi-hit process, including the production of galactose-deficient IgA1, anti-glycan response, formation and deposition of IgA1-containing immune complexes. The immune complexes deposition in glomerular mesangium activates the complement pathway, stimulates mesangial cells, and induces secretion of cytokines, chemokines, and extracellular matrix proteins resulting in tubulointerstitial inflammation and fibrosis $(29,30)$.
As indicated above, LN and IgAN shared immune-complex related pathogenesis, while the specific mechanisms remain different. Nevertheless, coexistence of IgAN and SLE was found in some cases (8-10). Genetic studies have pointed out partial sharing of mechanisms of LN and IgAN. Genes like CFH, HLA-DRA, HLA-DRB1, PXK, BLK, UBE2L3 and MTMR3 are shared loci between IgAN and SLE/LN based on GWAS, which highlights pathways including MHC class-II antigen presentation, complement regulation, signaling by the BCR, autophagy, and ubiquitin/proteasomedependent degradation shared between these two complex diseases $(18,27,31)$. As a result, identification between the LN and IgAN for patients with kidney injuries remains significant. As the marker of complement activation, C4d is involved into the pathogenesis of both $\mathrm{LN}$ and IgAN. However, studies comparing the C4d between LN and IgAN remain sparse (15-18). In this situation, our study aims to directly compare C4d in $\mathrm{LN}$ and IgAN from several aspects and explore whether C4d could act as an important marker for differential diagnosis between IgAN and LN.

In our study, we observed that $\mathrm{C} 4 \mathrm{~d}$ was a shared renal deposition molecule and distributed differently between LN and IgAN patients. It is indicated that LN and IgAN had comparable levels of serum C4d and urine C4d excretion. However, LN patients with renal C4d deposition were much more than that of IgAN patients, which is consistent with previous studies (15-17). Moreover, other studies further indicated that plasma $\mathrm{C} 4 \mathrm{~d}$ levels correlated with renal $\mathrm{C} 4 \mathrm{~d}$ deposition in LN patients (32). Mesangial deposition of C4d was found to be an independent predictor of urinary $\mathrm{C} 4 \mathrm{~d}$ levels for IgAN patients (33). We also revealed that the renal C4d distribution patterns differed between $\mathrm{LN}$ and IgAN patients. Compared with IgAN patients, the LN patients were more likely to have $\mathrm{C} 4 \mathrm{~d}$ deposition in renal glomerulus and less likely in tubules. Some other studies even explored the 
TABLE 6 | Univariate and multivariate Cox regression analysis for predictors of the disease relapse in LN patients.

\begin{tabular}{|c|c|c|c|c|c|c|}
\hline \multirow[t]{2}{*}{ Characteristics } & \multicolumn{3}{|c|}{ Univariate analysis } & \multicolumn{3}{|c|}{ Multivariate analysis } \\
\hline & HR & $95 \% \mathrm{Cl}$ & $P$ value & HR & $95 \% \mathrm{Cl}$ & $P$ value \\
\hline Age (years) & & & 0.280 & & & \\
\hline Gender(female vs. male) & & & 0.360 & & & \\
\hline UPE (g/d) & & & 0.095 & & & \\
\hline $\operatorname{Scr}(\mu \mathrm{mol} / \mathrm{L})$ & & & 0.361 & & & \\
\hline Serum albumin (g/L) & & & 0.191 & & & \\
\hline anti-dsDNA antibody (IU/mL) & 1.835 & $1.657-2.109$ & 0.002 & 1.105 & $1.002-1.365$ & 0.034 \\
\hline Urine C4d excretion ( $\mu \mathrm{g} / \mathrm{ml})$ & & & 0.125 & & & \\
\hline Serum C4d level $(\mu \mathrm{g} / \mathrm{ml})$ & & & 0.741 & & & \\
\hline Renal C4d deposition & 1.012 & $1.002-3.254$ & 0.003 & 1.007 & $1.003-1.054$ & 0.040 \\
\hline
\end{tabular}

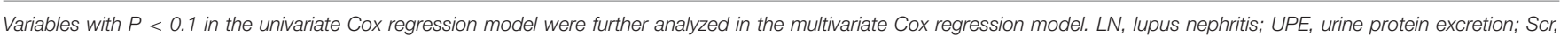
serum creatinine.

TABLE 7 | Univariate and multivariate Cox regression analysis for predictors of disease progression during the follow-up period for lgAN patients.

\begin{tabular}{|c|c|c|c|c|c|c|}
\hline \multirow[t]{2}{*}{ Characteristics } & \multicolumn{3}{|c|}{ Univariate analysis } & \multicolumn{3}{|c|}{ Multivariate analysis } \\
\hline & HR & $95 \% \mathrm{Cl}$ & $P$ value & HR & $95 \% \mathrm{Cl}$ & $P$ value \\
\hline Age (years) & & & 0.428 & & & \\
\hline Gender(female vs. male) & 0.139 & $0.017-1.111$ & 0.063 & & & \\
\hline UPE (g/d) & 1.221 & $1.062-1.404$ & 0.005 & & & \\
\hline $\operatorname{Scr}(\mu \mathrm{mol} / \mathrm{L})$ & 1.011 & $1.004-1.018$ & 0.003 & 1.010 & $1.020-1.030$ & 0.034 \\
\hline Serum albumin (g/L) & 0.860 & $0.760-0.960$ & 0.010 & & & \\
\hline Urine C4dexcretion ( $\mu \mathrm{g} / \mathrm{ml})$ & 8.095 & $2.529-25.912$ & $<0.001$ & & & \\
\hline Serum C4d level ( $\mu \mathrm{g} / \mathrm{ml})$ & 1.661 & $1.152-2.393$ & 0.007 & & & \\
\hline Renal C4d deposition & 2.011 & $1.990-5.210$ & 0.030 & 1.821 & $1.723-4.540$ & 0.040 \\
\hline
\end{tabular}

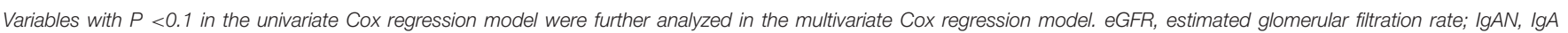
nephropathy; UPE, urine protein excretion; Scr, serum creatinine.

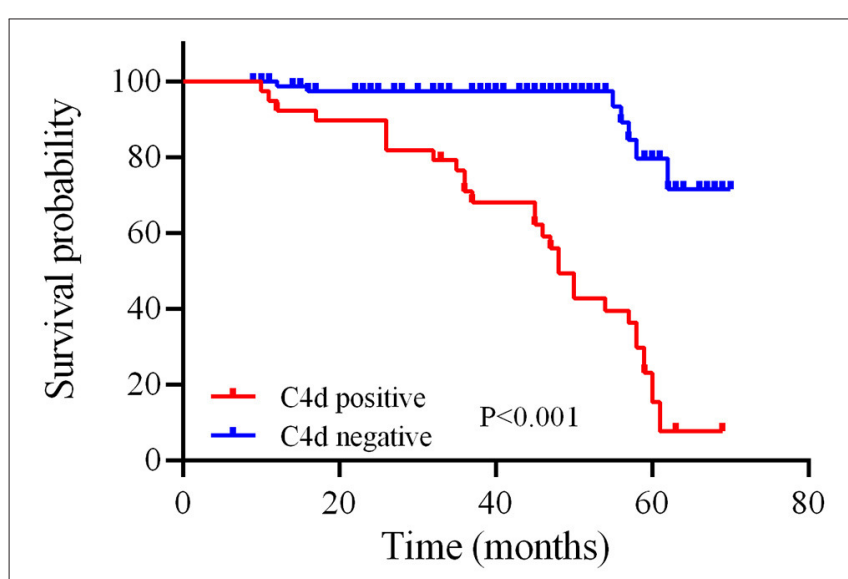

FIGURE 4 | Relationship between C4d deposition in renal tissue and disease progression by time of IgAN patients. The red and blue line separately illustrate the survival probability over time for IgAN patients with positive or negative renal C4d deposition. Kaplan-Meier analysis with log-rank test revealed a significant difference between groups $(P<0.001)$.

specific location of C4d in glomeruli and extraglomerular parts. Previous studies indicated that C4d deposition was mainly in glomerular mesangium for IgAN patients (34-36). For LN patients, the renal C4d deposition was predominantly along the glomerular capillary loops and to a lesser extent in mesangium $(15,34,37-39)$. Based on those findings, we could conclude that the differences and characters of renal C4d deposition pattern between $\mathrm{LN}$ and IgAN have made C4d a reliable screening and diagnostic tool to recognize and differentiate LN and IgAN.

In the present study, renal C4d deposition was especially strong in class $\mathrm{V}$ membranous $\mathrm{LN}$ and IgAN with tubulointerstitial fibrosis lesions. This result was similar with other studies. Kim et al. found a diffusely intense renal C4d deposition in class $\mathrm{V}$ membranous LN (15). For IgAN, tubulointerstitial fibrosis lesions were demonstrated to be the most consistent feature associated with renal C4d deposits in other studies $(18,40)$. Moreover, other studies also indicated that the renal $\mathrm{C} 4 \mathrm{~d}$ deposition significantly correlated with the presence of IgG, IgA, C3, C4, and C1q in $\operatorname{LN}$ patients $(37,38,41)$. In IgAN patients, glomerular $\mathrm{C} 4 \mathrm{~d}$ staining was found to be associated with mesangial IgM deposition (35). Based on those findings, it is suggested that C4d could also act as a factor to facilitate the recognition of worse pathologic features for LN and IgAN patients, which is beneficial for the therapeutic regimens to be adopted, thus promoting the clinical prognosis of the patients. 
It is demonstrated that LN patients with renal C4d deposition had a higher risk of relapse after treatment compared with the C4d negative patients. While there was no significant relationship between renal $\mathrm{C} 4 \mathrm{~d}$ deposition and $\mathrm{LN}$ progression. Ding et al. concluded that arteriolar C4d deposition was associated with worse renal outcomes, while C4d deposition in other renal compartments did not influence patient renal outcomes (39), which is instructive for our study. However, restricted by the fact that the cases in each subgroup of LN patients are too less to conduct the analysis, we cannot further confirm this conclusion. Moreover, it could also be attributed to the follow-up period, which was not long enough for LN patients to experience renal function deterioration. As previous studies have proved the definitive role of relapse with LN progression (42). Thus we could speculate that the relationship between renal C4d deposition and LN progression might be found when extending the followup duration.

In the present study, we demonstrated that renal $\mathrm{C} 4 \mathrm{~d}$ deposition is an independent predictor for IgAN progression during the follow-up period, which is similar with most of other studies indicated that C4d deposition was associated with lower eGFR, worse proteinuria, and worse renal survival for IgAN patients $(17,18,43-45)$. Previous studies indicated that renal survival at 5 and 20 years was 76 and $28 \%$ respectively for IgAN patients. In consideration of the followup period in our study, we finally selected a decline of eGFR by more than $20 \%$ or end-stage kidney disease as the indicators for the disease progression event. There was no significant correlation between renal C4d deposition and relapse after treatment for IgAN patients, which is different from LN patients.

There were also some limitations to this study. Firstly, the study population was under-represented in the general population. This is a single-center study. Bias in patient selection might therefore have been unavoidable. Secondly, restricted by the indications of renal biopsy, we had no access to renal tissues from healthy control, which make it difficult for us to explore C4d deposition in healthy kidneys. Moreover, the relationship between C4d stratified by the deposition sites in kidney, severity and kinds of pathologic lesions with clinical outcomes were not explored in this study as the patients with the onset of outcome events in each subgroup were too less to conduct the analysis. Finally, as

\section{REFERENCES}

1. Cameron JS. Lupus nephritis. J Am Soc Nephrol. (1999) 10:41324. doi: 10.1681/ASN.V102413

2. Sato N, Ohsawa I, Nagamachi S, Ishii M, Kusaba G, Inoshita $\mathrm{H}$, et al. Significance of glomerular activation of the alternative pathway and lectin pathway in lupus nephritis. Lupus. (2011) 20:1378-86. doi: 10.1177/0961203311415561

3. Liu T, Yang M, Xia Y, Jiang C, Li C, Jiang Z, et al. Microarray-based analysis of renal complement components reveals a therapeutic target for lupus nephritis. Arthritis Res Ther. (2021) 23:223. doi: 10.1186/s13075-021-02605-9 stated above, the longer follow-up period might contribute more valuable information.

\section{CONCLUSIONS}

In summary, we demonstrated that renal C4d deposition was shared between LN and IgAN patients, while the depositing proportion and patterns differed significantly between LN and IgAN. The renal C4d deposition was especially strong in class $\mathrm{V}$ membranous $\mathrm{LN}$ and $\operatorname{IgAN}$ with tubulointerstitial fibrosis lesions, meanwhile, acted as an independent predictor of relapse for $\mathrm{LN}$ patients and disease progression for IgAN patients.

\section{DATA AVAILABILITY STATEMENT}

The datasets presented in this article are not readily available as further analysis is going to be done based on it. Requests to access the datasets should be directed to Shan Mou, moushanrenji@126.com.

\section{ETHICS STATEMENT}

The studies involving human participants were reviewed and approved by Ethics Committee of Renji Hospital affiliated to Shanghai Jiao Tong University School of Medicine. The patients/participants provided their written informed consent to participate in this study.

\section{AUTHOR CONTRIBUTIONS}

SM, QW, and CQ: designed research. XY, YY, XS, HP, XC, LC, MZ, YX, ZN, CQ, QW, and SM: performed research. All authors contributed to the article and approved the submitted version.

\section{FUNDING}

This study was supported by Ministry of Science and Technology of China (2017YFE0110500), National Natural Science Foundation of China $(81770668,81970574)$. The study was also sponsored by Shanghai Municipal Health Commission (ZXYXZ-201904), Shanghai Jiao Tong University School of Medicine (18ZXY001) as well as Shanghai Outstanding Academic Leaders Plan. 
common diseases? J Clin Med. (2021) 10:4715. doi: $10.3390 / \mathrm{jcm} 102$ 04715

7. Suzuki H, Novak J. IgA glycosylation and immune complex formation in IgAN. Semin Immunopathol. (2021) 43:66978. doi: 10.1007/s00281-021-00883-8

8. Horino $\mathrm{T}$, Takao $\mathrm{T}$, Terada Y. IgA nephropathy in a patient with systemic lupus erythematosus. Lupus. (2010) 19:6504. doi: 10.1177/0961203309349384

9. Mac-Moune Lai F, Li EK, Tang NL, Li PK, Lui SF, Lai KN. IgA nephropathy: a rare lesion in systemic lupus erythematosus. Mod Pathol. (1995) 8:5-10.

10. Corrado A, Quarta L, Di Palma AM, Gesualdo L, Cantatore FP. IgA nephropathy in systemic lupus erythematosus. Clin Exp Rheumatol. (2007) 25:467-9.

11. Zhou XJ, Cheng FJ, Zhu L, Lv JC, Qi YY, Hou P, et al. Association of systemic lupus erythematosus susceptibility genes with IgA nephropathy in a Chinese cohort. Clin J Am Soc Nephrol. (2014) 9:788-97. doi: 10.2215/CJN.018 60213

12. Weinstein A, Alexander RV, Zack DJ. A review of complement activation in SLE. Curr Rheumatol Rep. (2021) 23:16. doi: 10.1007/s11926-021-0 0984-1

13. Wang H, Liu M. Complement C4, infections, and autoimmune diseases. Front Immunol. (2021) 12:694928. doi: 10.3389/fimmu.2021.694928

14. Feucht HE, Opelz G. The humoral immune response towards HLA class II determinants in renal transplantation. Kidney Int. (1996) 50:146475. doi: 10.1038/ki.1996.460

15. Kim MK, Maeng YI, Lee SJ, Lee IH, Bae J, Kang YN, et al. Pathogenesis and significance of glomerular $\mathrm{C} 4 \mathrm{~d}$ deposition in lupus nephritis: activation of classical and lectin pathways. Int J Clin Exp Pathol. (2013) 6:2157-67.

16. Shen Y, Chen XW, Sun CY, Dai M, Yan YC, Yang CD. Association between anti-beta2 glycoprotein I antibodies and renal glomerular C4d deposition in lupus nephritis patients with glomerular microthrombosis: a prospective study of 155 cases. Lupus. (2010) 19:1195-203. doi: 10.1177/09612033103 68409

17. Faria B, Henriques C, Matos AC, Daha MR, Pestana M, Seelen M. Combined C4d and CD3 immunostaining predicts immunoglobulin (Ig)A nephropathy progression. Clin Exp Immunol. (2015) 179:354-61. doi: 10.1111/cei. 12461

18. Espinosa M, Ortega R, Sánchez M, Segarra A, Salcedo MT, González $\mathrm{F}$, et al. Association of $\mathrm{C} 4 \mathrm{~d}$ deposition with clinical outcomes in $\operatorname{IgA}$ nephropathy. Clin J Am Soc Nephrol. (2014) 9:897-904. doi: 10.2215/CJN.097 10913

19. Radhakrishnan J, Cattran DC. The KDIGO practice guideline on glomerulonephritis: reading between the (guide)lines-application to the individual patient. Kidney Int. (2012) 82:840-56. doi: 10.1038/ki. 2012.280

20. The American College of Rheumatology response criteria for proliferative and membranous renal disease in systemic lupus erythematosus clinical trials. Arthritis Rheum. (2006) 54:421-32. doi: 10.1002/art. 21625

21. Kim JK, Kim JH, Lee SC, Kang EW, Chang TI, Moon SJ, et al. Clinical features and outcomes of IgA nephropathy with nephrotic syndrome. Clin J Am Soc Nephrol. (2012) 7:427-36. doi: 10.2215/CJN.04820511

22. Levey AS, Coresh J, Greene T, Marsh J, Stevens LA, Kusek JW, et al. Expressing the Modification of Diet in Renal Disease Study equation for estimating glomerular filtration rate with standardized serum creatinine values. Clin Chem. (2007) 53:766-72. doi: 10.1373/clinchem.2006.0 77180

23. Weening JJ, D’Agati VD, Schwartz MM, Seshan SV, Alpers CE, Appel GB, et al. The classification of glomerulonephritis in systemic lupus erythematosus revisited. J Am Soc Nephrol. (2004) 15:241-50. doi: 10.1097/01.ASN.0000108969.21691.5D

24. Trimarchi H, Barratt J, Cattran DC, Cook HT, Coppo R, Haas $M$, et al. Oxford Classification of IgA nephropathy 2016: an update from the IgA Nephropathy Classification Working Group. Kidney Int. (2017) 91:1014-21. doi: 10.1016/j.kint.2017. 02.003
25. O'Connor C, Fiuzat M, Mulder H, Coles A, Ahmad T, Ezekowitz JA, et al. Clinical factors related to morbidity and mortality in high-risk heart failure patients: the GUIDE-IT predictive model and risk score. Eur J Heart Fail. (2019) 21:770-8. doi: 10.1002/ejhf.1450

26. Cohen D, Colvin RB, Daha MR, Drachenberg CB, Haas M, Nickeleit V, et al. Pros and cons for C4d as a biomarker. Kidney Int. (2012) 81:62839. doi: 10.1038/ki.2011.497

27. Zhou XJ, Nath SK, Qi YY, Sun C, Hou P, Zhang YM, et al. Novel identified associations of RGS1 and RASGRP1 variants in IgA Nephropathy. Sci Rep. (2016) 6:35781. doi: 10.1038/srep35781

28. Yu F, Haas M, Glassock R, Zhao MH. Redefining lupus nephritis: clinical implications of pathophysiologic subtypes. Nat Rev Nephrol. (2017) 13:48395. doi: 10.1038/nrneph.2017.85

29. Magistroni R, D’Agati VD, Appel GB, Kiryluk K. New developments in the genetics, pathogenesis, and therapy of IgA nephropathy. Kidney Int. (2015) 88:974-89. doi: 10.1038/ki.2015.252

30. Roberts IS. Pathology of IgA nephropathy. Nat Rev Nephrol. (2014) 10:44554. doi: 10.1038/nrneph.2014.92

31. Zhu L, Zhai YL, Wang FM, Hou P, Lv JC, Xu DM, et al. Variants in Complement Factor $\mathrm{H}$ and Complement Factor H-Related Protein Genes, CFHR3 and CFHR1, affect complement activation in IgA nephropathy. J Am Soc Nephrol. (2015) 26:1195-204. doi: 10.1681/ASN.2014010096

32. Martin M, Trattner R, Nilsson SC, Björk A, Zickert A, Blom AM, et al. Plasma C4d Correlates With C4d Deposition in Kidneys and With Treatment Response in Lupus Nephritis Patients. Front Immunol. (2020) 11:582737. doi: 10.3389/fimmu.2020.582737

33. Segarra-Medrano A, Carnicer-Caceres C, Valtierra-Carmeno N, AgrazPamplona I, Ramos-Terrades N, Jatem Escalante E, et al. Study of the variables associated with local complement activation in IgA nephropathy. Nefrologia. (2017) 37:320-9. doi: 10.1016/j.nefro.2016.11.019

34. Chandra P. C4d in native glomerular diseases. Am J Nephrol. (2019) 49:8192. doi: 10.1159/000496059

35. Heybeli C, Unlu M, Yildiz S, Çavdar C, Sarioglu S, Camsari T. IgA nephropathy: association of C4d with clinical and histopathological findings and possible role of IgM. Ren Fail. (2015) 37:1464-9. doi: 10.3109/0886022X.2015.1077319

36. Rath A, Tewari R, Mendonca S, Badwal S, Nijhawan VS. Oxford classification of IgA nephropathy and C4d deposition; correlation and its implication. $J$ Nephropharmacol. (2016) 5:75-9.

37. Kim SH, Jeong HJ. Glomerular C4d deposition indicates in situ classic complement pathway activation, but is not a marker for lupus nephritis activity. Yonsei Med J. (2003) 44:75-80. doi: 10.3349/ymj.2003.44.1.75

38. Batal I, Liang K, Bastacky S, Kiss LP, McHale T, Wilson NL, et al. Prospective assessment of C4d deposits on circulating cells and renal tissues in lupus nephritis: a pilot study. Lupus. (2012) 21:13-26. doi: 10.1177/0961203311422093

39. Ding Y, Yu X, Wu L, Tan Y, Qu Z, Yu F. The Spectrum of C4d deposition in renal biopsies of lupus nephritis patients. Front Immunol. (2021) 12:654652. doi: 10.3389/fimmu.2021.654652

40. Worawichawong S, Plumworasawat S, Liwlompaisan W, Sumethkul V, Phakdeekitcharoen B, Udomsubpayakul U, et al. Distribution pattern of mesangial C4d deposits as predictor of kidney failure in IgA nephropathy. PLoS ONE. (2021) 16:e0252638. doi: 10.1371/journal.pone.02 52638

41. Li SJ, Liu ZH, Zen CH, Wang QW, Wang Y, Li LS. Peritubular capillary C4d deposition in lupus nephritis different from antibody-mediated renal rejection. Lupus. (2007) 16:875-80. doi: 10.1177/0961203307083279

42. Mejía-Vilet JM, Córdova-Sánchez BM, Arreola-Guerra JM, MoralesBuenrostro LE, Uribe-Uribe NO, Correa-Rotter R. Renal flare prediction and prognosis in lupus nephritis Hispanic patients. Lupus. (2016) 25:31524. doi: 10.1177/0961203315606985

43. Nasri H, Ahmadi A, Rafieian-Kopaei M, Bashardoust B, Nasri P, Mubarak M. Association of glomerular C4d deposition with various demographic data in IgA nephropathy patients; a preliminary study. J Nephropathol. (2015) 4:19-23. doi: 10.12860/jnp.2015.04

44. Fabiano RCG, de Almeida Araújo S, Bambirra EA, Oliveira EA, Simões ESAC, Pinheiro SVB. Mesangial C4d deposition may predict progression of kidney 
disease in pediatric patients with IgA nephropathy. Pediatr Nephrol. (2017) 32:1211-20. doi: 10.1007/s00467-017-3610-y

45. Segarra A, Romero K, Agraz I, Ramos N, Madrid A, Carnicer C, et al. Mesangial C4d Deposits in Early IgA Nephropathy. Clin J Am Soc Nephrol. (2018)13:258-64. doi: 10.2215/CJN.02530317

Conflict of Interest: The authors declare that the research was conducted in the absence of any commercial or financial relationships that could be construed as a potential conflict of interest.

Publisher's Note: All claims expressed in this article are solely those of the authors and do not necessarily represent those of their affiliated organizations, or those of the publisher, the editors and the reviewers. Any product that may be evaluated in this article, or claim that may be made by its manufacturer, is not guaranteed or endorsed by the publisher.

Copyright (c) 2022 Yang, Yuan, Shao, Pang, Che, Cao, Zhang, Xu, Ni, Qi, Wang and Mou. This is an open-access article distributed under the terms of the Creative Commons Attribution License (CC BY). The use, distribution or reproduction in other forums is permitted, provided the original author(s) and the copyright owner(s) are credited and that the original publication in this journal is cited, in accordance with accepted academic practice. No use, distribution or reproduction is permitted which does not comply with these terms. 